\title{
La toxicidad de la política española en Twitter durante la pandemia de la COVID-19
}

\author{
Frederic Guerrero-Solé \\ Universitat Pompeu Fabra \\ frederic.guerrero@upf.edu \\ https://orcid.org/0000-0001-8145-8707
}

\author{
Olivier PhilipPe \\ Universitat Pompeu Fabra \\ olivier.philippe@upf.edu \\ https://orcid.org/0000-0003-3180-2025
}

\section{The toxicity of Spanish politics in Twitter during the COVID-19 pandemics}

\begin{abstract}
RESUMEN
La toxicidad de los mensajes políticos es una creciente amenaza a la confianza que los ciudadanos depositan en el sistema democrático. En particular, las redes sociales son lugares en los que esta toxicidad es más evidente. La pandemia de la COVID-19 y el estado de alarma decretado por el gobierno español parece el contexto ideal para el aumento de la toxicidad en el discurso político. Este artículo analiza la toxicidad de los mensajes de los diputados del Congreso español en Twitter durante el estado de alarma a partir de la herramienta Jigsaw de Google (Conversation Al by conversationai, s. f.; Wulczyn et al., 2017). También se analizan los 15 días anteriores y posteriores a este estado de alarma. El estudio concluye que el periodo del estado de alarma no se caracterizó por un aumento de la toxicidad de los mensajes de los diputados. Observamos, sin embargo, diferencias significativas entre partidos políticos. La extrema derecha de Vox fue el partido más tóxico, seguido por el PP. Finalmente, el estudio también muestra que los políticos hombres tien-

den a publicar más mensajes tóxicos que las mujeres.
\end{abstract}

PALABRAS CLAVE

COVID-19; Toxicidad; Twitter, Comunicación política; Diputados; España

\section{ABSTRACT}

The toxicity of political messages is a growing threat to the trust that citizens place in the democratic system. In particular, social networks are platforms in which toxicity is more evident. The COVID-19 pandemic and the state of alarm decreed by the Spanish government seems the ideal context for the increase of toxicity in political discourse. This article analyses the toxicity of the messages of the deputies of the Spanish Congress on Twitter during the state of alarm using the Google Jigsaw tool (Conversation Al by conversationai, s. f.; Wulczyn et al., 2017). The 15 days before and after this state of alarm are also analysed. The study concludes that the period of the alarm state was not characterized by an increase in the toxicity of the messages of the deputies. However, we observe significant differences between political parties. Vox's extreme right was the most toxic party, followed by the PP. Finally, the study also shows that male politicians tend to post more toxic messages than women.

\section{KEYWORDS}

COVID-19; Toxicity; Twitter; Political communication; Deputies; Spain.

Hipertext.net, política de Ciencia Abierta | Acceso abierto a los datos de la investigación (share research data) Enlace al DATASET de la investigación: https://repositori.upf.edu/handle/10230/45911 


\section{Introducción}

\subsection{El impacto de la COVID-19 en España}

La pandemia del coronavirus SARS-COV-2 (COVID-19) ha tenido un enorme impacto en las dinámicas de funcionamiento de las democracias y de los sistemas de medios a nivel global (Casero-Ripollés, 2020). En particular, ha afectado al consumo de información y, en el caso particular de los Estados Unidos, el mayor incremento fue el experimentado en el consumo de noticias a través de redes sociales (Casero-Ripollés, 2020). Una de las consecuencias de este incremento de la información consumida a través de las redes sobre la pandemia ha sido, según Aleixandre-Benavent, Castelló-Cogollos y Valderrama-Zurián (2020) la sobreinformación y la consecuente difusión de noticias falsas. Otra de las consecuencias de la crisis de la COVID-19 ha sido el aumento de la toxicidad de los mensajes publicados en las redes sociales, en particular en Twitter. Según un estudio publicado por el Reuters Institute de la Universidad de Oxford (Majó-Vázquez, et al., 2020) el porcentaje de mensajes tóxicos que fueron publicados en Twitter durante la pandemia de COVID-19 fue de 21\%, con picos de hasta el $25 \%$ en los momentos más críticos de la pandemia.

El debate político en los medios de comunicación se considera una de las bases de las democracias occidentales. También el debate en redes sociales como Twitter, en la que se ha demostrado que, con el tiempo, ha habido un incremento de la polarización política y de los mensajes incívicos (Majó-Vázquez, et al., 2020). En particular, en una democracia representativa el tono del discurso de los políticos elegidos democráticamente es fundamental en el proceso de interpretación por parte de la ciudadanía de lo que se entiende por debate político. De este modo, la inclusión de un lenguaje tóxico por parte de los políticos puede repercutir en una imagen negativa de la acción política y acentuar la polarización entre diferentes grupos ideológicos de la sociedad. Por ello, el seguimiento de la toxicidad en las redes, y más aún en circunstancias tan extraordinarias como las derivadas de la crisis del COVID-19, es de gran importancia para medir cuál es la salud de nuestra democracia.

Este artículo tiene como objetivo analizar la toxicidad de los mensajes publicados por los diputados del Congreso en Twitter durante el estado de alarma, y 15 días antes y después de este estado de alarma. Para ello, utilizamos la herramienta Jigsaw (Conversation Al by conversationai, s. f.; Wulczyn et al., 2017) de análisis de la toxicidad de los textos, utilizada anteriormente en el trabajo publicado por Majó-Vázquez (2020).

\subsection{El discurso tóxico}

Más allá de las definiciones del discurso del odio (hate speech), definido como aquel lenguaje de menosprecio a personas o colectivos en función de su raza, género, religión u otros (Nockleby, 2000), el lenguaje tóxico se refiere a aquellas palabras utilizadas para denigrar a un colectivo o adversario político, entre las cuales están los insultos, el lenguaje grosero, la falta de respeto, o el uso de palabras malsonantes que, en un principio, no forman parte del lenguaje propiamente político. Tal y como se recoge en el trabajo de Majó-Vázquez et al. (2020), Wulczyn et al. (2017) los mensajes tóxicos se definen como aquellos que incluyen comentarios inadmisibles, groseros, e irrespetuosos. Son mensajes de menosprecio que forman parte de los que denominamos discurso o lenguaje tóxico que, o bien invitan al resto de usuarios a abandonar la discusión (Wulczyn et al. 2017), o bien al uso de un lenguaje al mismo nivel (espiral tóxica).

Siguiendo la estela determinista de Postman (1985) y Meyrowitz (1985), Ott (2016) considera que Twitter privilegia el discurso simple, irreflexivo e irrespetuoso que infecta, como un cáncer, el debate social. En particular, Ott (2016) defiende que el discurso de Twitter es irrespetuoso porque su registro es informal, y porque despersonaliza las interacciones sociales. El presidente norteamericano, Donald Trump, es según este autor un ejemplo paradigmático de este estilo discursivo, al que se le atribuye parte de su éxito electoral en 2016. Este tipo de discurso irrespetuoso, que denominamos tóxico, está relacionado con el discurso del odio (Nockleby, 2000), una de las principales líneas de investigación en el análisis de contenido de redes sociales en la última década (Watanabe, Bouazizi y Ohtsuki, 2018). La implementación de herramientas para la detección automática de discurso del odio (Anzovino, Fersini y Rosso, 2018; Lee, Lee, Park y Han, 2018; Pereira-Kohatsu, Quijano-Sánchez, Liberatore y Camacho-Collados, 2019) o de los ataques personales (Wulczyn, Thain y Dixon, 2017) es una de las áreas que más se ha desarrollado. Sin embargo, tal y como apunta Bruns (2019), tras el escándalo de Cambridge Analytica en 2018, las redes sociales han restringido el acceso a sus datos a los investigadores, hecho que dificulta el análisis de cuestiones de gran importancia como el lenguaje abusivo, el acoso, el discurso del odio o las campañas de desinformación.

Esta investigación analiza la toxicidad de los mensajes políticos en Twitter durante el estado de alarma causado por la pandemia de la COVID-19 en España. Para 
ello, hemos utilizado una muestra de los mensajes publicados por 273 de los 350 diputados del Congreso español entre el 14 de marzo y el 21 de junio de 2020, y 15 días antes y después de este periodo. Teniendo en cuenta lo expuesto, las preguntas de investigación son las siguientes:

RQ1: ¿Cuál es el nivel de toxicidad de los mensajes publicados por los diputados del Congreso durante el estado de alarma? ¿Y antes y después del estado de alarma?

RQ1.1. ¿Hay diferencias significativas en cuanto al grado de toxicidad entre partidos políticos?

Considerando que la variable género puede intervenir en el nivel de toxicidad de los mensajes, de la misma manera que interviene en el número de ataques y mensajes incívicos que las diputadas pueden recibir a través de Twitter (Southern y Harmer, 2019), nos preguntamos finalmente lo siguiente:

RQ2. ¿Hay diferencias significativas en la proporción de toxicidad de los mensajes de los diputados españoles durante la pandemia en función de su género?

\section{Método y muestra}

La muestra analizada fue recogida a diario utilizando el acceso Twitter REST API (Standard Search API, s. f.) entre el 28 de febrero y el 6 de julio de 2020. Este periodo comprende el estado de alarma, así como 15 días antes y 15 después de este estado de alarma. Durante este periodo se recogieron todos los tuits publicados por los diputados electos al Congreso de los Diputados español que tenían cuenta de Twitter. En total, 273 usuarios fueron monitorizados y clasificados en función del partido político al que representan (Tabla 1).

En total, se recogieron 428,610 (incluyendo retuits). Estos tuits fueron procesados para reducir el tamaño de la muestra.

De este modo, el símbolo de retuit (RT), las menciones, las URLs y el símbolo \# fueron eliminados del contenido de los tuits. Además, se llevó a cabo un procesamiento

\begin{tabular}{|c|l|l|}
\hline Partido & Número de tuits & Número de usuarios \\
\hline Cs & 12,078 & 9 \\
\hline Otros & 16,298 & 33 \\
\hline PP & 69,060 & 72 \\
\hline PSOE & 64,855 & 90 \\
\hline UP & 15,450 & 32 \\
\hline VOX & 62,642 & 37 \\
\hline
\end{tabular}

Tabla 1. Número de usuarios y de tuits publicados durante el periodo analizado por partido político. Elaboración propia. clásico para cualquier tarea de análisis de Procesamiento de Lenguaje Natural (NLP). Todas las palabras fueron transformadas en su equivalente en minúsculas. Posteriormente, utilizando la librería Gensim (Řehůřek y Sojka, 2010), se omitieron las palabras vacías (stop words) (Rajaraman y Ullman, 2011) en español. Del mismo modo, todos los tuits con menos de 5 palabras (después de borrar símbolos y palabras vacías) fueron eliminados de la muestra.

El método de detección de toxicidad depende de cada lengua, así que sólo los tuits redactados en español fueron considerados. Para la detección de la lengua, usamos la librería fasttext de Facebook (Joulin et al., 2016), que genera una medida entre 0 y 1 asociada al lenguaje detectado. En nuestra investigación, mantuvimos en la muestra aquellos tuits con una medida igual o superior a 0.6. Después de estas fases de procesamiento, la muestra final estaba compuesta de 240,383 tuits (retuits incluidos). Mantuvimos los retuits por el hecho de ser, por lo general, muestras de aprobación (Guerrero-Solé, 2017; 2018) que forman parte de la estrategia comunicativa de los diputados.

\subsection{Detección de la toxicidad}

Tal y como se ha mencionado anteriormente, entendemos por lenguaje tóxico aquel que incluye comentarios inadmisibles, groseros, e irrespetuosos, que pueden llevar a alguien a abandonar una discusión (Fortuna y Nunes, 2018, p. 8). Para los autores, este lenguaje puede ir desde el lenguaje abusivo hasta las blasfemias y los insultos.

Para el análisis de la toxicidad hemos utilizado la API Jigsaw (Conversation Al by conversationai, s. f.; Wulczyn et al., 2017) que ya ha sido utilizado previamente para detectar la toxicidad en comunidades en línea (Miller et al., 2017; Mittos et al., s. f.), así como durante la pandemia de COVID-19 en Twitter (Majó-Vázquez et al., 2020). Esta API puede detectar y cuantificar la toxicidad de un texto en 6 lenguas diferentes, entre ellas el español. Esta API devuelve una puntuación entre 0 y 1 asociada a cada tuit, indicando su nivel de toxicidad. Esta medida refleja el porcentaje de codificadores humanos que consideran un mensaje como tóxico, y representa la probabilidad de que un mensaje sea considerado como tóxico.

\section{Resultados}

Lo primero que calculamos fue la distribución de los mensajes en función de su toxicidad. Como podemos ver en la Figura 1, la mayoría de los tuits tienen un nivel bajo o muy bajo de toxicidad. En esta investigación, asumimos que un mensaje es tóxico a partir de una pun- 


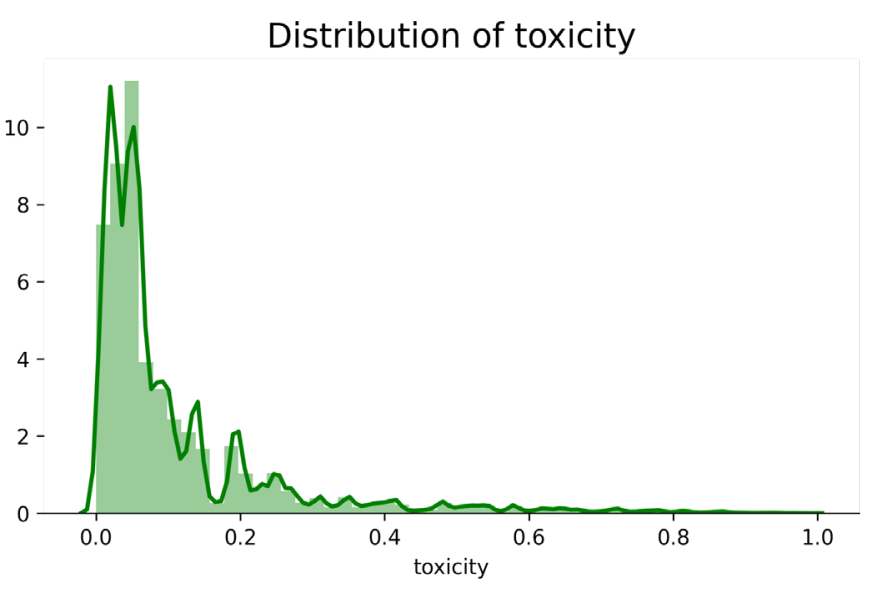

Figura 1. Distribución de la toxicidad en la muestra de mensajes publicados por los diputados españoles entre el 28 de febrero y el 6 de julio de 2020. Elaboración propia.

tuación de 0.4. De este modo, cualquier mensaje por debajo de 0.4 se considera no tóxico. El porcentaje final de tuits considerados como tóxicos fue del 5,12\% (11,724 mensajes).

\subsection{La evolución temporal de la toxicidad}

A partir del número de tuits tóxicos, medimos la media diaria y semanal para analizar cuál fue la evolución temporal de la toxicidad (Figura 2). La línea naranja representa la media de la frecuencia de tuits tóxicos por semana, mientras que la línea azul es la media diaria. El histograma en gris representa el total de tuits publicados por día, sean tóxicos o no.

\begin{tabular}{|c|l|l|l|}
\hline Periodo & \multicolumn{1}{|c|}{$\begin{array}{c}\text { Mensajes } \\
\text { no tóxicos }\end{array}$} & $\begin{array}{c}\text { Mensajes } \\
\text { tóxicos }\end{array}$ & $\begin{array}{c}\text { Proporción } \\
\text { de toxicidad }\end{array}$ \\
\hline $\begin{array}{c}\text { 1. Pre- Estado } \\
\text { de alarma }\end{array}$ & 23,543 & 1464 & 5.85 \\
\hline $\begin{array}{c}\text { 2. Estado de } \\
\text { alarma }\end{array}$ & 188,512 & 9402 & 4.75 \\
\hline $\begin{array}{c}\text { 3. Post- Estado } \\
\text { de alarma }\end{array}$ & 16,604 & 858 & 4.91 \\
\hline
\end{tabular}

Tabla 2. Proporción de mensajes tóxicos en los diferentes periodos analizados. Elaboración propia.

\subsection{La evolución de la toxicidad antes, durante y después del estado de alarma}

En la Tabla 2, podemos observar las diferencias entre el periodo anterior (del 28 de febrero al 13 de marzo), durante (del 14 de marzo al 21 de junio) y posterior (del 22 de junio al 06 de julio) al confinamiento.

A continuación, se realizó una prueba chi-cuadrado para saber si la diferencia en la proporción de toxicidad entre los diferentes periodos era estadísticamente significativa. La prueba dio como resultado $X^{2}(2,240382)$ $=58.361, p<0.00 .1$, por lo que la diferencia fue, efectivamente, estadísticamente significativa.

\subsection{La toxicidad por partidos políticos}

A continuación, calculamos la proporción de toxicidad por partido político durante el mismo periodo (Figura 3).

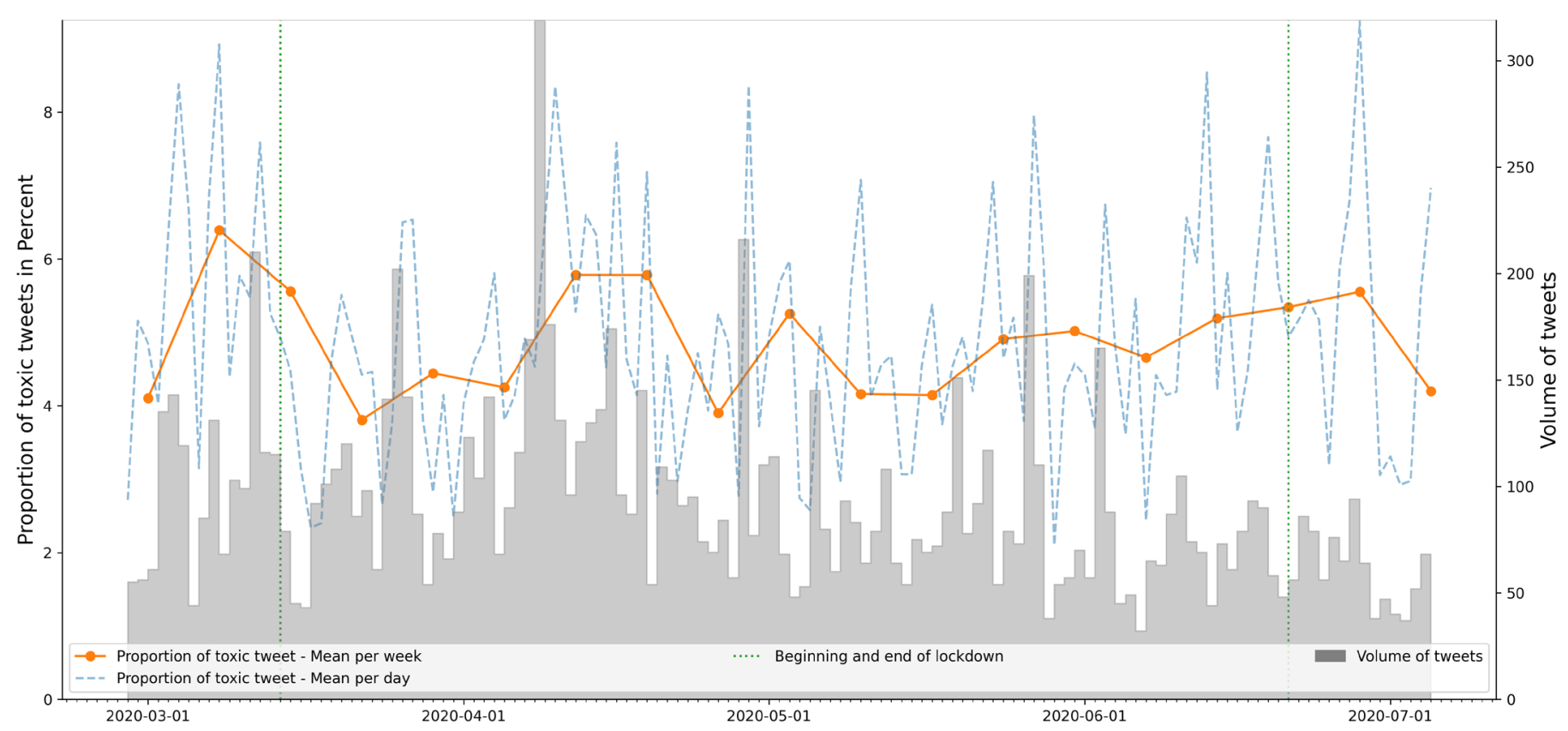

Figura 2. Evolución diaria (línea de puntos azul) y semanal (línea naranja) de los tuits tóxicos publicados por los diputados españoles entre el 28 de febrero y el 6 de julio de 2020. Elaboración propia. 


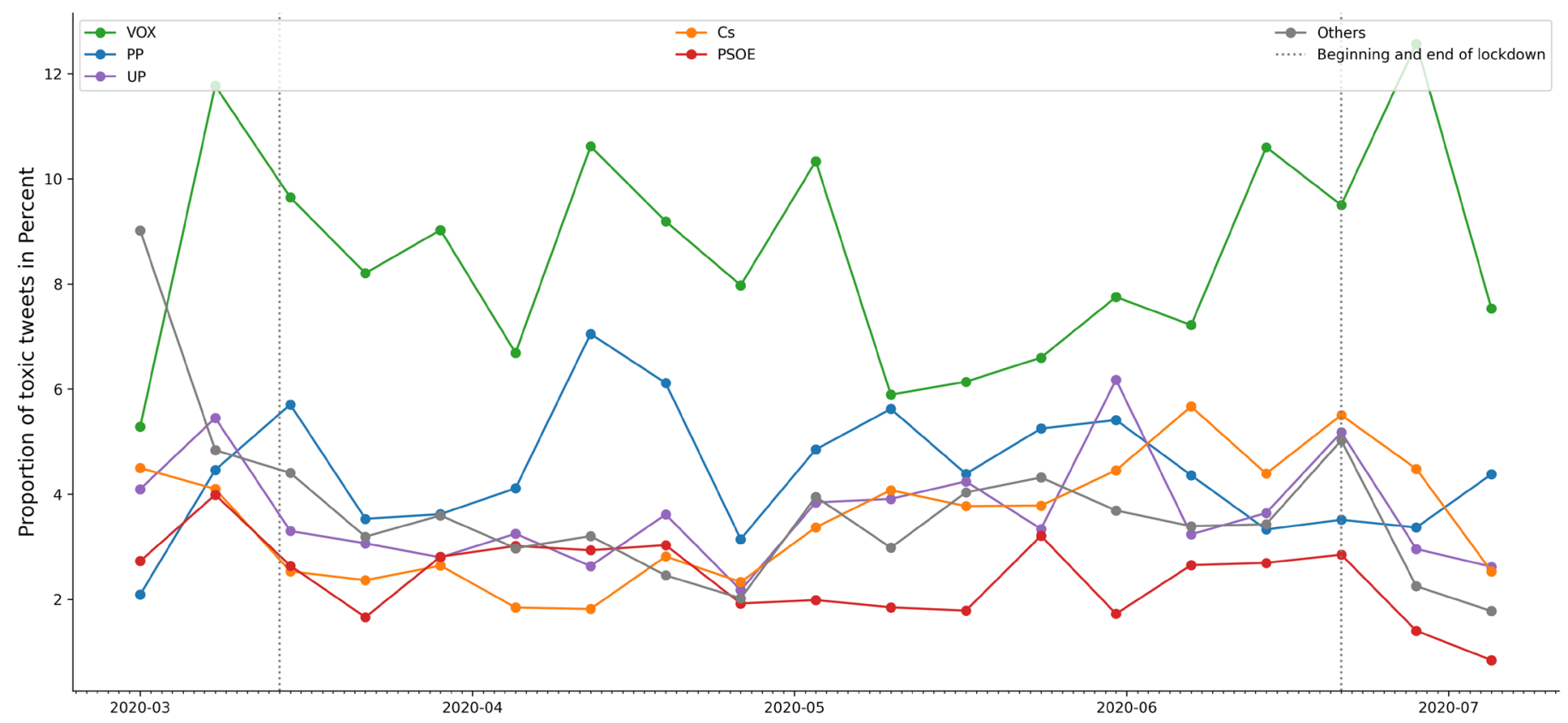

Figura 3. Evolución semanal de los tuits tóxicos publicados por los diferentes partidos políticos españoles entre el 28 de febrero y el 6 de julio de 2020. Elaboración propia.

Los partidos políticos mantienen un nivel muy similar de toxicidad durante los 15 días anteriores al estado de alarma, excepto en el caso de UP, que es ligeramente superior al resto. Sin embargo, a partir de la declaración del estado de alarma, todos los partidos parecen reducir la toxicidad de sus mensajes publicados en Twitter, excepto Vox y PP. En general, Vox es el partido que publica mensajes más tóxicos. Al final del estado de alarma, Vox incrementa su toxicidad, mientras que Cs releva al PP en la segunda posición. Finalmente, en los 15 días posteriores al final del estado de alarma, el PP incrementa el volumen de mensajes tóxicos, mientras que el resto de partidos se mantienen estables., o incluso disminuyen.

\subsection{Diferencias en la proporción de toxicidad entre partidos durante el estado de alarma}

Aplicamos las mismas técnicas para observar las diferencias entre partidos en la toxicidad de los mensajes

\begin{tabular}{|c|l|l|l|}
\hline Partido & $\begin{array}{c}\text { Mensajes no } \\
\text { tóxicos }\end{array}$ & \multicolumn{1}{|c|}{$\begin{array}{c}\text { Mensajes } \\
\text { tóxicos }\end{array}$} & $\begin{array}{c}\text { Proporción de } \\
\text { toxicidad }\end{array}$ \\
\hline VOX & 46,412 & 4,144 & 8.20 \\
\hline PP & 54,729 & 2,702 & 4.70 \\
\hline UP & 12,107 & 444 & 3.54 \\
\hline Cs & 9,272 & 331 & 3.45 \\
\hline Otros & 12,454 & 443 & 3.43 \\
\hline PSOE & 53,538 & 1,338 & 2.44 \\
\hline
\end{tabular}

Tabla 3. Proporción de mensajes tóxicos de los diferentes partidos durante el estado de alarma. Elaboración propia. publicados por sus diputados durante el estado de alarma (Tabla 3).

Realizamos un test chi-cuadrado para examinar las diferencias en la frecuencia de toxicidad entre partidos políticos, obteniendo una significación estadística $X^{2}$ (5, $N=197914)=2101.937, p<.001$.

\subsection{Diferencias en la toxicidad por género del diputado}

Finalmente, calculamos el nivel de toxicidad de los mensajes publicados por los diputados en función de su género. En la muestra, identificamos a 152 hombres y 121 mujeres. En la tabla 4 podemos observar la diferencia en la proporción de toxicidad agregada por géneros. Realizamos un test de chi-cuadrado y comprobamos que las diferencias de toxicidad por género eran estadísticamente significativas, $X^{2}(1, N=240383)=123.777, p$ $<.001$.

\section{Conclusiones}

Este artículo ha analizado el nivel de toxicidad de los mensajes publicados por los diputados españoles durante el estado de alarma decretado por el gobierno

\begin{tabular}{|c|l|l|l|}
\hline Género & No tóxico & Tóxico & Proporción de toxicidad \\
\hline Hombre & 132,949 & 7,426 & 5.29 \\
\hline Mujer & 95,710 & 4,298 & 4.30 \\
\hline
\end{tabular}

Tabla 4. Toxicidad de los mensajes publicados por los diputados por género. Elaboración propia. 
de Pedro Sánchez. A través del análisis de la toxicidad con la herramienta Jigsaw utilizada en diversos trabajos anteriores (Wulczyn et al., 2017; Miller et al., 2017; Mittos et al., s. f.), hemos podido dar respuesta a las preguntas de investigación planteadas. Por lado, hemos concluido que el porcentaje mensajes tóxicos es relativamente bajo (5,12\% del total de la muestra), comparado con los resultados de Majó-Vázquez et al. (2020). Debemos observar que, a diferencia de la muestra de Majó-Vázquez et al. (2020), este trabajo analiza mensajes publicados por representantes electos, hecho que podría explicar la diferencia en las proporciones de toxicidad.

En cuanto a la evolución temporal de la toxicidad antes, durante y después del estado de alarma decretado por el gobierno español, nuestro estudio concluye que la toxicidad de los diputados españoles se redujo durante el estado de alarma, en comparación con la toxicidad antes y después. Esta diferencia es estadísticamente significativa. Nuestro estudio también concluye que hay diferencias muy evidentes en los porcentajes de toxicidad en función del partido político de los diputados. Así, nuestro estudio concluye que el partido de ultraderecha Vox fue el que más mensajes tóxicos publicó, en particular durante el estado de alarma. Las diferencias observadas con el resto de partidos políticos fueron muy destacadas (la proporción de mensajes tóxicos de Vox fue de 8.20, mientras que la del PP, segundo partido más tóxico, fue de 4.70). Además, Cs, UP y Otros mostraron unas proporciones muy similares. Finalmente, nuestro estudio concluye que el PSOE partido mayoritario del gobierno español, fue el que menor proporción de toxicidad mostró en sus mensajes en Twitter.

Finalmente, analizamos el nivel de toxicidad de los mensajes publicados por los diputados en función de su género. Los resultados nos muestran que la proporción de mensajes tóxicos publicados por diputados hombres es significativamente mayor que en el caso de las mujeres. Serán necesarias investigaciones futuras que nos muestren cuáles son las causas de esta diferencia.

Esta investigación añade conocimiento al estudio de la toxicidad de los mensajes políticos en España. Teniendo en cuenta que la toxicidad del debate político tiene consecuencias negativas en la percepción que la ciudadanía tiene de la política y los políticos (Majó-Vázquez et al., 2020), así como en misma polarización política, creemos que esta es una línea de investigación con un largo recorrido futuro. La creciente polarización política, que se visualiza en las redes sociales y, en particular, en Twitter, y la presencia cada vez mayor de la ultraderecha en la política española sugiere un incremento de la toxicidad en los mensajes políticos. En particular, y atendiendo a los resultados de este estudio, entre los políticos de extrema derecha y las comunidades políticas afines. La toxicidad puede ser interpretada, en este sentido, como una estrategia más de la extrema derecha para minar la confianza en la democracia representativa y sugerir la necesidad de formas más radicales y autoritarias de gobierno.

Finalmente, añadir que el análisis muestra una menor toxicidad de los mensajes durante el estado de alarma. Este escenario, sin embargo, puede cambiar mucho en el futuro post-COVID-19, teniendo en cuenta las crisis sociales, económicas y políticas que pueden derivarse de la crisis sanitaria.

\section{Financiación}

Esta investigación ha contado con la financiación del proyecto 'Polarización, duplicación de las audiencias activas y populismo en Twitter. Análisis de la influencia de los discursos populistas en los debates políticos en España (2016-2020)' (DATAPOP), por parte del Ministerio de Ciencia, Innovación y Universidades de España. PGC2018-097352-A-I00.

\section{Referencias}

Aleixandre-Benavent, R., Castelló-Cogollos, L., Valderrama-Zurián, J. C. (2020). Información y comunicación durante los primeros meses de COVID-19. Infodemia, desinformación y papel de los profesionales de la información. El profesional de la información, 29, (4), e290408. https://doi.org/10.3145/epi.2020.jul.08

Anzovino, M., Fersini, E., y Rosso, P. (2018). Automatic identification and classification of misogynistic language on Twitter. En Lecture Notes in Computer Science (including subseries Lecture Notes in Artificial Intelligence and Lecture Notes in Bioinformatics). https://doi. org/10.1007/978-3-319-91947-8_6

Bruns, A. (2019). After the 'APIcalypse': social media platforms and their fight against critical scholarly research. Information Communication and Society. https://doi.org/10.1080/1369118X. 2019.1637447

Burnap, P., y Williams, M. L. (2016). Us and them: identifying cyber hate on Twitter across multiple protected characteristics. EPJ Data Science. https://doi.org/10.1140/epjds/s13688-016-0072-6

Casero-Ripollés, A. (2020). Impact of COVID-19 on the media system. Communicative and democratic consequences of news consumption during the outbreak. El profesional de la información, 29(2), e290223. https://doi.org/10.3145/epi.2020.mar.23

Conversation Al by conversationai. (s. f.). https://conversationai. github.io/

Guerrero-Solé, F. (2017). Community Detection in Political Discussions on Twitter: An Application of the Retweet Overlap Network Method to the Catalan Process Toward Independence. Social Science Computer Review, 35(2), 244-261. https://doi.org/10.1177/0894439315617254

Guerrero-Solé, Frederic. (2018). Interactive Behavior in Political Discussions on Twitter: Politicians, Media, and Citizens' Patterns of 
Interaction in the 2015 and 2016 Electoral Campaigns in Spain. Social Media + Society. https://doi.org/10.1177/2056305118808776

Nockleby, J. T. (2000). Hate Speech, volume 1. Encyclopedia of the American Constitution (2nd ed., edited by Leonard W. Levy, Kenneth L. Karst et al. Macmillan.

Lee, H. S., Lee, H. R., Park, J. U., y Han, Y. S. (2018). An abusive text detection system based on enhanced abusive and non-abusive word lists. Decision Support Systems. https://doi.org/10.1016/j. dss.2018.06.009

Majó-Vázquez, S., Nielsen, R. K., Verdú, J., Rao, N., de Domenico, M., y Papaspiliopoulos, O. (2020). Volume and Patterns of Toxicity in Social Media Conversations during the COVID-19 Pandemic. Reuters Institute. https://reutersinstitute.politics.ox.ac.uk/volume-and-patterns-toxicity-social-media-conversations-during-covid-19-pandemic

Meyrowitz, J. (1985). No sense of place: The impact of electronic media on social behavior. Oxford University Press.

Miller, M., Banerjee, T., Muppalla, R., Romine, W., y Sheth, A. (2017). What Are People Tweeting About Zika? An Exploratory Study Concerning Its Symptoms, Treatment, Transmission, and Prevention. JMIR Public Health and Surveillance, 3(2), e38. https://doi.org/10.2196/ publichealth.7157

Mittos, A., Zannettou, S., Blackburn, J., y Cristofaro, E. D. (s. f.). "And We Will Fight For Our Race!" A Measurement Study of Genetic Testing Conversations on Reddit and 4chan. https://arxiv.org/pdf/1901.09735. pdf

Ott, B. L. (2017). The age of Twitter: Donald J. Trump and the politics of debasement. Critical Studies in Media Communication. https://doi. org/10.1080/15295036.2016.1266686

Pereira-Kohatsu, J. C., Quijano-Sánchez, L., Liberatore, F., y Camacho-Collados, M. (2019). Detecting and monitoring hate speech in twitter. Sensors (Switzerland). https://doi.org/10.3390/s19214654

Postman, N. (1985). Amusing ourselves to death: Public discourse in the age of show business. Penguin Books.

Rajaraman, A., y Ullman, J. (2011). Data Mining. En Mining of Massive Datasets (pp. 1-17). Cambridge University Press. https://doi. org/10.1017/CBO9781139058452.002

Southern, R., y Harmer, E. (2019). Twitter, Incivility and "Everyday" Gendered Othering: An Analysis of Tweets Sent to UK Members of Parliament. Social Science Computer Review. https://doi. org/10.1177/0894439319865519

Terkourafi, M., Catedral, L., Haider, I., Karimzad, F., Melgares, J., Mostacero-Pinilla, C., ... Weissman, B. (2018). Uncivil Twitter: A sociopragmatic analysis. Journal of Language Aggression and Conflict. https:// doi.org/10.1075/jlac.00002.ter

Watanabe, H., Bouazizi, M., y Ohtsuki, T. (2018). Hate Speech on Twitter: A Pragmatic Approach to Collect Hateful and Offensive Expressions and Perform Hate Speech Detection. IEEE Access. https://doi.org/10.1109/ACCESS.2018.2806394

Wulczyn, E., Thain, N., y Dixon, L. (2017). Ex machina: Personal attacks seen at scale. En 26th International World Wide Web Conference, WWW 2017. https://doi.org/10.1145/3038912.3052591

\section{CV}

Frederic Guerrero-Solé. Es profesor de Sociología de la Comunicación y de Métodos Cuantitativos de Investigación en Comunicación, e investigador del departamento de comunicación de la Universidad Pompeu Fabra de Barcelona. Sus principales líneas de investigación son la comunicación política, las redes sociales y los efectos de los medios. Ha publicado diversos trabajos sobre comunicación política en Twitter en revista como Information, Communication and Society, Social Science Computer Review o Social Media + Society. Actualmente coordina el grupo de investigación POLCOM de la UPF, y es el investigador principal del proyecto DATAPOP sobre populismo y polarización política en redes sociales.

Olivier Philippe. Ha realizado un máster psicología y sociología en la Universidad de Lieja, Bélgica, así como un máster y doctorado en ciencias de la web en la Universidad de Southampton, Reino Unido. Empezó a trabajar en el Software Sustainability Institute en junio de 2015 como investigador de políticas y fue responsable del aspecto técnico y de investigación del equipo de investigación de políticas. Sus principales tareas fueron el soporte en la recopilación y análisis de datos para comprender la comunidad de Research Software Engineer. Su trabajo consistió en construir encuestas a nivel nacional e internacional. También ha desarrollado herramientas en Python para ayudar a diferentes proyectos en la recopilación de datos, así como desarrollar proyectos con modelos de aprendizaje automático. Desde febrero de 2020 forma parte del equipo de investigación DATAPOP de la UPF. Sus principales tareas son el análisis de datos y el desarrollo de herramientas para el análisis de la comunicación política en redes sociales. 